\title{
ON PSEUDO GENERALIZED QUASI-EINSTEIN MANIFOLDS
}

\author{
A. A. SHAIKH AND SANJIB KUMAR JANA
}

Abstract. The object of the present paper is to introduce a type of non-flat Riemannian manifold called pseudo generalized quasi-Einstein manifold and studied some properties of such a manifold with several non-trivial examples.

\section{Introduction}

In 2000 M. C. Chaki and R. K. Maity [1] introduced the notion of quasi-Einstein manifold. A non-flat Riemannian manifold $\left(M^{n}, g\right)(n>2)$ is said to be quasi-Einstein manifold if its Ricci tensor $S$ of type $(0,2)$ is not identically zero and satisfies the following:

$$
S(X, Y)=\alpha g(X, Y)+\beta A(X) A(Y)
$$

where $\alpha, \beta$ are scalars such that $\beta \neq 0$ and $A$ is a non-zero 1 -form defined by $g(X, U)=A(X)$ for all vector fields $X$; $U$ being a unit vector field, called the generator of the manifold. An $n$-dimensional manifold of this kind is denoted by $(Q E)_{n}$. The scalars $\alpha, \beta$ are known as the associated scalars.

Recently U. C. De and G. C. Ghosh [3] introduced the notion of generalized quasi-Einstein manifold. A non-flat Riemannian manifold $\left(M^{n}, g\right)(n>2)$ is said to be generalized quasiEinstein manifold if its Ricci tensor $S$ of type $(0,2)$ is not identically zero and satisfies the following condition:

$$
S(X, Y)=\alpha g(X, Y)+\beta A(X) A(Y)+\gamma B(X) B(Y)
$$

where $\alpha, \beta$ and $\gamma$ are non-zero scalars and $A, B$ are non-zero 1-forms defined respectively by $g(X, U)=A(X)$ and $g(X, V)=B(X)$ for all vector fields $X$. The unit vector fields $U$ and $V$ corresponding to the 1 -forms $A$ and $B$ are orthogonal i.e., $g(U, V)=0$. Also $U$ and $V$ are known as the generators of the manifold. Such an $n$-dimensional manifold of this kind is denoted by $G(Q E)_{n}$.

The present paper deals with a non-flat Riemannian manifold called pseudo generalized quasi-Einstein manifold.

Received May 1, 2006.

2000 Mathematics Subject Classification. 53B50, 53C25, 53C35..

Key words and phrases. Pseudo generalized quasi-Einstein manifold, pseudo generalized quasi-constant curvature, harmonic vector field, viscous fluid spacetime. 
A Riemannian manifold $\left(M^{n}, g\right)(n>2)$ is called a pseudo generalized quasi-Einstein manifold if its Ricci tensor $S$ of type $(0,2)$ is not identically zero and satisfies the following:

$$
S(X, Y)=\alpha g(X, Y)+\beta A(X) A(Y)+\gamma B(X) B(Y)+\delta D(X, Y)
$$

where $\alpha, \beta, \gamma$ and $\delta$ are non-zero scalars and $A, B$ are non-zero 1-forms such that

$$
g(X, U)=A(X), \quad g(X, V)=B(X)
$$

for all vector fields $X ; U, V$ being mutually orthogonal unit vector fields called the generators of the manifold, $D$ is a symmetric $(0,2)$ tensor, with zero trace, which satisfies the condition

$$
D(X, U)=0
$$

for all vector fields $X$. Also $\alpha, \beta, \gamma$ and $\delta$ are called the associated scalars; $A, B$ are the associated 1-forms of the manifold and $D$ is called the structure tensor of the manifold. Such an $n$-dimensional manifold will be denoted by $P(G Q E)_{n}$.

Section 2 is concerned with the preliminaries and it is shown that the scalars $\alpha+\beta$ and $\alpha+\gamma+\delta D(V, V)$ are the Ricci curvatures along the directions of the vector fields $U$ and $V$ respectively. After preliminaries, in section 3, we prove the existence theorem for a $P(G Q E)_{n}$. Section 4 is devoted to the conformally flat $P(G Q E)_{n}$ and introduced the notion of pseudo generalized quasi-constant curvature. It is shown that a manifold of pseudo generalized quasiconstant curvature is a $P(G Q E)_{n}$. But the converse is not true, in general. However a $P(G Q E)_{3}$ is a manifold of pseudo generalized quasi-constant curvature. Section 5 deals with some geometric properties of $P(G Q E)_{n}$. In section 6 we investigate the application of $P(G Q E)_{4}$ to the general relativistic viscous fluid spacetime admitting heat flux and it is shown that such a spacetime obeying Einstein's equation with a cosmological constant is a connected semiRiemannian $P(G Q E)_{4}$. In the last section we discover several non-trivial examples of the $P(G Q E)_{n}$ which are neither $(Q E)_{n}$ nor $G(Q E)_{n}$.

\section{Preliminaries}

We consider a $P(G Q E)_{n}(n>2)$. Let $\left\{e_{i}: i=1, \ldots, n\right\}$ be an orthonormal basis of the tangent space at any point of the manifold. Then setting $X=Y=e_{i}$ in (1.3) and taking summation over $i, 1 \leq i \leq n$ we obtain

$$
r=n \alpha+\beta+\gamma,
$$

where $r$ is the scalar curvature of the manifold. Also from (1.3) we have

$$
\begin{gathered}
S(U, U)=\alpha+\beta, \\
S(V, V)=\alpha+\gamma+\delta D(V, V) \text { and } \\
S(U, V)=0 .
\end{gathered}
$$

It is known that if $P$ be a unit vector field, then $S(P, P)$ is the Ricci curvature in the direction of $P$. Hence from (2.2) and (2.3) we can state the following: 
Theorem 2.1. In a $P(G Q E)_{n}(n>2)$, the scalars $\alpha+\beta$ and $\alpha+\gamma+\delta D(V, V)$ are the Ricci curvatures in the directions of the generators $U$ and $V$ respectively.

Let $Q$ and $L$ be the symmetric endomorphisms of the tangent space at any point of the manifold corresponding to the Ricci tensor $S$ and the structure tensor $D$ respectively i.e., $g(Q X, Y)=S(X, Y)$ and $g(L X, Y)=D(X, Y)$. Further, let $s^{2}$ and $d^{2}$ denote the squares of the length of the Ricci tensor $S$ and the structure tensor $D$ respectively. Then $s^{2}=\sum_{i=1}^{n} S\left(Q e_{i}, e_{i}\right)$ and $d^{2}=\sum_{i=1}^{n} D\left(L e_{i}, e_{i}\right)$. Now from (1.3) we get

$$
\sum_{i=1}^{n} S\left(Q e_{i}, e_{i}\right)=(n-2) \alpha^{2}+(\alpha+\beta)^{2}+(\alpha+\gamma)^{2}+\gamma \delta D(V, V)+\delta \sum_{i=1}^{n} S\left(L e_{i}, e_{i}\right) .
$$

Also from (1.3) we obtain

$$
\sum_{i=1}^{n} S\left(L e_{i}, e_{i}\right)=\gamma D(V, V)+\delta \sum_{i=1}^{n} D\left(L e_{i}, e_{i}\right)
$$

Hence from (2.5) and (2.6) it follows that

$$
s^{2}=n \alpha^{2}+\beta^{2}-\gamma^{2}+2[\alpha \beta+\gamma S(V, V)]+\delta^{2} d^{2} .
$$

From (2.7) it follows that $\delta>\frac{s}{d}$ (resp. $<,=$ ) according as $n \alpha^{2}+\beta^{2}-\gamma^{2}+2[\alpha \beta+\gamma S(V, V)]<0$ (resp. $>,=$ ). Hence we can state the following:

Theorem 2.2. In a $P(G Q E)_{n}(n>2)$ the associated scalar $\delta$ is less than or equal or greater than the ratio which the length of the Ricci tensor $S$ bears to the length of the structure tensor $D$ according as $n \alpha^{2}+\beta^{2}-\gamma^{2}+2[\alpha \beta+\gamma S(V, V)]>0$ or, $=0$ or,$<0$ respectively.

\section{Existence Theorem of $P(G Q E)_{n}(n>2)$}

To prove the existence theorem of $P(G Q E)_{n}$ ( $\left.n>2\right)$, we first state a well-known result ([5], [6]) as follows:

Proposition 3.1. For a connected orientable manifold $M^{n}$ the following assertions are equivalent:

1. There is a non-vanishing vector field $V$ on $M^{n}$.

2. Either $M^{n}$ is non-compact, or $M^{n}$ is compact and has Euler number $\chi\left(M^{n}\right)=0$.

Theorem 3.1. Let $\left(M^{n}, g\right)$ be a connected orientable Riemannian manifold which is either non-compact or compact with vanishing Euler number. If the Ricci tensor $S$ of type $(0,2)$ of a Riemannian manifold is non-vanishing and satisfies the following relation

$$
\begin{aligned}
S(Y, Z) S(X, W)-S(X, Z) S(Y, W)= & p_{1}[g(Y, Z) g(X, W)-g(X, Z) g(Y, W)] \\
& +p_{2} g(T X, W) g(Y, Z)
\end{aligned}
$$


where $p_{1}, p_{2}$ are non-zero scalars and $T$ is the symmetric endomorphism of the tangent space at any point of the manifold corresponding to a tensor field of type $(0,2)$, then the manifold is a pseudo generalized quasi-Einstein manifold.

Proof. From the Proposition 3.1, it follows that there is a non-vanishing vector field $V$ on the manifold $\left(M^{n}, g\right)$ under consideration such that $g(X, V)=B(X)$ for all vector fields $X$. Then setting $Y=Z=V$ in (3.1) yields

$$
\begin{aligned}
S(V, V) S(X, W)-S(X, V) S(W, V)= & p_{1}[g(V, V) g(X, W)-g(X, V) g(W, V)] \\
& +p_{2} g(T X, W) g(V, V),
\end{aligned}
$$

which can be written as

$$
\begin{aligned}
a S(X, W)-B(Q X) B(Q W)= & p_{1}\|V\|^{2} g(X, W)-p_{1} B(X) B(W) \\
& +p_{2}\|V\|^{2} g(T X, W)
\end{aligned}
$$

where $a=S(V, V)$ and $B(Q X)=g(Q X, V)=S(X, V)$. Since $S(V, V)$ is the Ricci curvature in the direction of the generator $V$ and the Ricci tensor is non-vanishing, it follows that the scalar $a$ is non-vanishing. From (3.2) it follows that

$$
S(X, W)=\alpha g(X, W)+\beta A(X) A(W)+\gamma B(X) B(W)+\delta D(X, W)
$$

where $\alpha=\frac{p_{1}\|V\|^{2}}{a}, \beta=\frac{1}{a}, \gamma=-\frac{p_{1}}{a}, \delta=\frac{p_{2}\|V\|^{2}}{a}, A(X)=B(Q X)$ and $D(X, W)=g(T X, W)$. Since $V$ is non-null, $S \neq 0, p_{1}$ and $p_{2}$ are non-zero scalars, it follows that $\alpha, \beta, \gamma, \delta$ are non-zero scalars. Hence the manifold is a $P(G Q E)_{n}$.

\section{Conformally flat $P(G Q E)_{n}(n>3)$}

Let $R$ be the curvature tensor of type $(0,4)$ of a conformally flat $P(G Q E)_{n}$. Then we have

$$
\begin{aligned}
R(X, Y, Z, W)= & \frac{1}{n-2}[g(Y, Z) S(X, W)-g(X, Z) S(Y, W) \\
& +g(X, W) S(Y, Z)-g(Y, W) S(X, Z)] \\
& -\frac{r}{(n-1)(n-2)}[g(Y, Z) g(X, W)-g(X, Z) g(Y, W)],
\end{aligned}
$$

where $r$ is the scalar curvature of the manifold. Using (1.3) and (2.1) in (4.1) we obtain

$$
\begin{aligned}
R(X, Y, Z, W)= & \frac{\alpha(n-2)-\beta-\gamma}{(n-1)(n-2)}[g(Y, Z) g(X, W)-g(X, Z) g(Y, W)] \\
& +\frac{\beta}{n-2}[g(Y, Z) A(X) A(W)-g(X, Z) A(Y) A(W) \\
& +g(X, W) A(Y) A(Z)-g(Y, W) A(X) A(Z)] \\
& +\frac{\gamma}{n-2}[g(Y, Z) B(X) B(W)-g(X, Z) B(Y) B(W) \\
& +g(X, W) B(Y) B(Z)-g(Y, W) B(X) B(Z)] \\
& +\frac{\delta}{n-2}[g(Y, Z) D(X, W)-g(X, Z) D(Y, W) \\
& +g(X, W) D(Y, Z)-g(Y, W) D(X, Z)] .
\end{aligned}
$$


According to Chen and Yano [2], a Riemannian manifold $\left(M^{n}, g\right)(n \geq 3)$ is said to be of quasi-constant curvature if it is conformally flat and its curvature tensor $R$ of type $(0,4)$ has the form

$$
\begin{aligned}
R(X, Y, Z, W)= & a_{1}[g(Y, Z) g(X, W)-g(X, Z) g(Y, W)] \\
& +a_{2}[g(Y, Z) A(X) A(W)-g(X, Z) A(Y) A(W) \\
& +g(X, W) A(Y) A(Z)-g(Y, W) A(X) A(Z)],
\end{aligned}
$$

where $A$ is a 1 -form and $a_{1}, a_{2}$ are scalars of which $a_{2} \neq 0$.

Also according to De and Ghosh [3], a Riemannian manifold $\left(M^{n}, g\right)(n \geq 3)$ is said to be of generalized quasi-constant curvature if it is conformally flat and its curvature tensor $R$ of type $(0,4)$ has the form

$$
\begin{aligned}
R(X, Y, Z, W)= & b_{1}[g(Y, Z) g(X, W)-g(X, Z) g(Y, W)] \\
& +b_{2}[g(Y, Z) A(X) A(W)-g(X, Z) A(Y) A(W) \\
& +g(X, W) A(Y) A(Z)-g(Y, W) A(X) A(Z)] \\
& +b_{3}[g(Y, Z) B(X) B(W)-g(X, Z) B(Y) B(W) \\
& +g(X, W) B(Y) B(Z)-g(Y, W) B(X) B(Z)],
\end{aligned}
$$

where $A$ and $B$ are 1 -forms and $b_{1}, b_{2}, b_{3}$ are non-zero scalars. Generalizing this notion we define the manifold of pseudo generalized quasi-constant curvature as follows:

A Riemannian manifold $\left(M^{n}, g\right)(n \geq 3)$ is said to be of pseudo generalized quasi-constant curvature if it is conformally flat and its curvature tensor $R$ of type $(0,4)$ satisfies the condition

$$
\begin{aligned}
R(X, Y, Z, W)= & \alpha_{1}[g(Y, Z) g(X, W)-g(X, Z) g(Y, W)] \\
& +\alpha_{2}[g(Y, Z) A(X) A(W)-g(X, Z) A(Y) A(W) \\
& +g(X, W) A(Y) A(Z)-g(Y, W) A(X) A(Z)] \\
& +\alpha_{3}[g(Y, Z) B(X) B(W)-g(X, Z) B(Y) B(W) \\
& +g(X, W) B(Y) B(Z)-g(Y, W) B(X) B(Z)] \\
& +\alpha_{4}[g(Y, Z) D(X, W)-g(X, Z) D(Y, W) \\
& +g(X, W) D(Y, Z)-g(Y, W) D(X, Z)],
\end{aligned}
$$

where $\alpha_{1}, \alpha_{2}, \ldots, \alpha_{4}$ are non-zero scalars and $D$ is a symmetric tensor of type $(0,2)$. Now the relation (4.2) can be written as

$$
\begin{aligned}
R(X, Y, Z, W)= & \beta_{1}[g(Y, Z) g(X, W)-g(X, Z) g(Y, W)] \\
& +\beta_{2}[g(Y, Z) A(X) A(W)-g(X, Z) A(Y) A(W) \\
& +g(X, W) A(Y) A(Z)-g(Y, W) A(X) A(Z)] \\
& +\beta_{3}[g(Y, Z) B(X) B(W)-g(X, Z) B(Y) B(W) \\
& +g(X, W) B(Y) B(Z)-g(Y, W) B(X) B(Z)] \\
& +\beta_{4}[g(Y, Z) D(X, W)-g(X, Z) D(Y, W) \\
& +g(X, W) D(Y, Z)-g(Y, W) D(X, Z)],
\end{aligned}
$$


where $\beta_{1}=\frac{\alpha(n-2)-\beta-\gamma}{(n-1)(n-2)}, \beta_{2}=\frac{\beta}{n-2}, \beta_{3}=\frac{\gamma}{n-2}$ and $\beta_{4}=\frac{\delta}{n-2}$ are non-zero scalars. Comparing (4.3) and (4.4), it follows that the manifold is of pseudo generalized quasi-constant curvature. This leads to the following:

Theorem 4.1. A conformally flat $P(G Q E)_{n}(n>3)$ is a manifold of pseudo generalized quasi-constant curvature.

Let us now consider a manifold of pseudo generalized quasi-constant curvature. Then from (4.3) it follows that

$$
S(X, Y)=\bar{\alpha} g(X, Y)+\bar{\beta} A(X) A(Y)+\bar{\gamma} B(X) B(Y)+\bar{\delta} D(X, Y),
$$

where $\bar{\alpha}=(n-1) \alpha_{1}+\alpha_{2}+\alpha_{3}, \bar{\beta}=(n-2) \alpha_{2}, \bar{\gamma}=(n-2) \alpha_{3}$ and $\bar{\delta}=(n-2) \alpha_{4}$ are non-zero scalars. Thus we have the following:

Theorem 4.2. A manifold $\left(M^{n}, g\right)(n>2)$ of pseudo generalized quasi-constant curvature is a $P(G Q E)_{n}$.

Now a $P(G Q E)_{n}$ is not a manifold of pseudo generalized quasi-constant curvature in general. However, since a 3-dimensional Riemannian manifold is conformally flat, it follows by virtue of Theorem 4.1 that a $P(G Q E)_{3}$ is a manifold of pseudo generalized quasi-constant curvature. This leads to the following:

Corollary 4.1. $A P(G Q E)_{3}$ is a manifold of pseudo generalized quasi-constant curvature.

\section{Geometric Properties of $P(G Q E)_{n}(n>2)$}

This section deals with some geometric properties of $P(G Q E)_{n}(n>2)$. From (1.3) it follows that

$$
S(X, U)=(\alpha+\beta) g(X, U) \text { for all } X .
$$

This leads to the following:

Theorem 5.1. In a $P(G Q E)_{n}(n>2)$ the generator $U$ is an eigenvector of the Ricci tensor $S$ corresponding to the eigen value $\alpha+\beta$.

Next we suppose that in a $P(G Q E)_{n}(n>2)$, the generator $U$ is a parallel vector field. Then we have $\nabla_{X} U=0$ for all $X$, which implies that $R(X, Y) U=0$ and hence $S(X, U)=0$ for all $X$. Again from (1.3) we have

$$
S(X, U)=(\alpha+\beta) A(X) .
$$

Therefore we must have $\alpha+\beta=0$. Thus we have the following:

Theorem 5.2. If the generator $U$ of a $P(G Q E)_{n}(n>2)$ is a parallel vector field then the associated scalars $\alpha, \beta$ are related by $\alpha+\beta=0$. 
Again since $U$ and $V$ are orthogonal unit vector fields, we have from (2.2) that

$$
g(Q U, U)=\alpha+\beta,
$$

which implies that $Q U$ is orthogonal to $U$ if and only if $\alpha+\beta=0$. Hence we can state the following:

Theorem 5.3. In a $P(G Q E)_{n}(n>2), Q U$ is orthogonal to $U$ if and only if $\alpha+\beta=0$.

Further from (2.3) we obtain

$$
g(Q V, V)=\alpha+\gamma+\delta D(V, V)
$$

which implies that $Q V$ is orthogonal to $V$ if and only if $\alpha+\gamma+\delta D(V, V)=0$. Thus we have the following:

Theorem 5.4. In a $P(G Q E)_{n}(n>2), Q V$ is orthogonal to $V$ if and only if $\alpha+\gamma+\delta D(V, V)=$ 0 .

We now consider a compact orientable $P(G Q E)_{n}(n>2)$ without boundary. From (1.3) we have

$$
S(X, X)=\alpha g(X, X)+\beta A(X) A(X)+\gamma B(X) B(X)+\delta D(X, X) .
$$

Let us assume that $\theta_{U}$ be the angle between $U$ and any vector field $X ; \theta_{V}$ be the angle between $V$ and any vector field $X$. Therefore, $\cos \theta_{U}=\frac{g(X, U)}{\sqrt{g(X, X)}}$ and $\cos \theta_{V}=\frac{g(X, V)}{\sqrt{g(X, X)}}$. Further, we assume that $\theta_{U} \geq \theta_{V}$. Then we have $\cos \theta_{V} \geq \cos \theta_{U}$ and consequently $g(X, V) \geq g(X, U)$. Hence from (5.1) we have

$$
S(X, X) \geq(\alpha+\beta+\gamma)\{g(X, U)\}^{2} \text { when } \alpha, \beta, \gamma, \delta D(X, X) \text { are positive. }
$$

Definition 5.1. A vector field $H$ in a Riemannian manifold $\left(M^{n}, g\right)(n>2)$ is said to be harmonic [7] if

$$
d \tau=0 \quad \text { and } \quad \delta \tau=0
$$

where $\tau(X)=g(X, H)$ for all $X$.

It is known from [7] that in a compact, orientable Riemannian manifold $\left(M^{n}, g\right)(n>2)$, the following relation holds

$$
\int_{M=P(G Q E)_{n}}\left[S(X, X)-\frac{1}{2}|d \tau|^{2}+|\nabla X|^{2}-(\delta \tau)^{2}\right] d v=0 \quad \text { for any vector field } X,
$$

where ' $d v$ ' denotes the volume element of $M$. Now let $X \in \chi(M)$ be a harmonic vector field. Then (5.4) yields by virtue of (5.3) that

$$
\int_{M}\left[S(X, X)+|\nabla X|^{2}\right] d v=0 \quad \text { for any vector field } X .
$$


Hence if each of $\alpha, \beta, \gamma, \delta D(X, X)$ of $P(G Q E)_{n}$ are positive, then (5.2) and (5.5) together yields

$$
\int_{M}\left[(\alpha+\beta+\gamma)|g(X, U)|^{2}+|\nabla X|^{2}\right] d v \leq 0,
$$

which implies by virtue of $\alpha+\beta+\gamma>0$ that

$$
g(X, U)=0 \text { and } \nabla X=0 \text { for any vector field } X .
$$

Thus from (5.6), it follows that $X$ is orthogonal to $U$ and $X$ is a parallel vector field.

Similarly for the case, $\theta_{U} \leq \theta_{V}$, arguing as before it can be shown that $g(X, V)=0$ and $\nabla X=0$ for any vector field $X$. Thus we can state the following:

Theorem 5.5. In a compact, orientable $P(G Q E)_{n}(n>2)$ without boundary any harmonic vector field $X$ is parallel and orthogonal to one of the generators of the manifold which makes greatest angle with the vector $X$ provided that $\alpha, \beta, \gamma$ and $\delta D(X, X)$ are positive scalars.

\section{General relativistic viscous fluid spacetime admitting heat flux}

Let $\left(M^{4}, g\right)$ be a connected semi-Riemannian viscous fluid spacetime admitting heat flux and satisfying Einstein's equation with a cosmological constant $\lambda$. Also let $U$ be the unit timelike velocity vector field of the fluid, $V$ be the unit heat flux vector field and $D$ be the anisotropic pressure tensor of the fluid. Then we have

$$
\begin{gathered}
g(U, U)=-1, \quad g(V, V)=1, \quad g(U, V)=0, \\
D(X, Y)=D(Y, X), \quad \operatorname{Tr} . D=0, \quad D(X, U)=0 \text { for all vector fields } X .
\end{gathered}
$$

Let

$$
g(X, U)=A(X), \quad g(X, V)=B(X) \text { for all vector field } X .
$$

Also let $T$ be the energy-momentum tensor of type $(0,2)$ describing the matter distribution of such a fluid and it be of the following form [4]

$$
T(X, Y)=p g(X, Y)+(\sigma+p) A(X) A(Y)+B(X) B(Y)+D(X, Y)
$$

where $\sigma$ and $p$ are the energy density and isotropic pressure respectively. General relativity flows from Einstein's equation given by

$$
S(X, Y)-\frac{r}{2} g(X, Y)+\lambda g(X, Y)=k T(X, Y)
$$

for all vector fields $X, Y$, where $S$ is the Ricci tensor of type $(0,2), r$ is the scalar curvature, $\lambda$ is a cosmological constant. Thus by virtue of (6.4), the above equation can be written as

$$
\begin{aligned}
S(X, Y)-\frac{r}{2} g(X, Y)+\lambda g(X, Y)= & k[p g(X, Y)+(\sigma+p) A(X) A(Y) \\
& +B(X) B(Y)+D(X, Y)] .
\end{aligned}
$$


Let us now consider a $P(G Q E)_{4}$ viscous fluid spacetime with the generator $U$ as the flow vector field of the fluid.

Again from (6.5) we have

$$
S(X, Y)=\left[k p+\frac{r}{2}-\lambda\right] g(X, Y)+k(\sigma+p) A(X) A(Y)+k B(X) B(Y)+k D(X, Y)
$$

which shows that the spacetime under consideration is a $P(G Q E)_{4}$ with $k p+\frac{r}{2}-\lambda, k(\sigma+p)$, $k$ and $k$ as associated scalars; $A$ and $B$ as associated 1-forms; $U$ and $V$ as generators and $D$ as the structure tensor of type $(0,2)$. Hence we can state the following:

Theorem 6.1. A viscous fluid spacetime admitting heat flux and satisfying Einstein's equation with a cosmological constant is a 4-dimensional connected semi-Riemannian pseudo generalized quasi-Einstein manifold.

Using (1.3) and (2.1) in (6.5) we get

$$
\begin{aligned}
\frac{2 k p-2 \lambda+2 \alpha+\beta+\gamma}{2} g(X, Y)= & {[\beta-k(\sigma+p)] A(X) A(Y) } \\
& +(\gamma-k) B(X) B(Y)-k D(X, Y) .
\end{aligned}
$$

Setting $Y=U$ in (6.6) we obtain by virtue of (6.1)-(6.3) that

$$
\frac{2 k p-2 \lambda+2 \alpha+\beta+\gamma}{2} A(X)=[k \sigma+k p-\beta] A(X) \text { for all vector field } X .
$$

Again setting $X=U$ in (6.7) we obtain

$$
\sigma=\frac{2 \alpha+3 \beta+\gamma-2 \lambda}{2 k}
$$

Now contracting (6.5) we get

$$
r-2 r+4 \lambda=k(3 p-\sigma+1),
$$

which yields by virtue of (2.1) and (6.8) that

$$
p=\frac{6 \lambda-6 \alpha+\beta-\gamma-2 k}{6 k} .
$$

Since $\alpha, \beta, \gamma$ are not constants, from (6.8) and (6.9) it follows that $\sigma$ and $p$ are not constants. Hence we can state the following:

Theorem 6.2. If a viscous fluid $P(G Q E)_{4}$ spacetime admitting heat flux obeys Einstein's equation with a cosmological constant then none of the energy density and isotropic pressure of the fluid can be a constant.

Now if the associated scalars $\alpha, \beta, \gamma$ are constants then it follows from (6.8) and (6.9) that $\sigma$ and $p$ are constants. Since $\sigma>0$ and $p>0$, we have from (6.8) and (6.9) that

$$
\lambda<\frac{2 \alpha+3 \beta+\gamma}{2} \text { and } \lambda>\frac{6 \alpha-\beta+\gamma-2 k}{6}
$$


and hence

$$
\frac{6 \alpha-\beta+\gamma-2 k}{6}<\lambda<\frac{2 \alpha+3 \beta+\gamma}{2} .
$$

Thus we have the following:

Theorem 6.3. If a viscous fluid $P(G Q E)_{4}$ spacetime admitting heat flux obeys Einstein's equation with a cosmological constant $\lambda$, then $\lambda$ satisfies the relation (6.10).

\section{Some examples of $P(G Q E)_{n}$}

This section deals with several examples of $P(G Q E)_{n}$. On the real number space $R^{n}$ (with coordinates $x^{1}, x^{2}, \ldots, x^{n}$ ) we define a suitable Riemannian metric $g$ such that $R^{n}$ becomes a Riemannian manifold $\left(M^{n}, g\right)$. We calculate the components of the Ricci tensor and then we verify the defining condition (1.3).

Example 1. We define a Riemannian metric on the 4-dimensional real number space $R^{4}$ by the formula

$$
\begin{aligned}
d s^{2}= & g_{i j} d x^{i} d x^{j}=\left(d x^{1}\right)^{2}+\left(x^{1}\right)^{2}\left(d x^{2}\right)^{2}+\left(x^{1} \sin x^{2}\right)^{2}\left(d x^{3}\right)^{2}+\left(d x^{4}\right)^{2}, \\
& (i, j=1,2, \ldots, 4),
\end{aligned}
$$

where $x^{1} \neq 0$ and $0<x^{2}<\frac{\pi}{2}$. Then the only non-vanishing components of the Christoffel symbols and the curvature tensor are

$$
\begin{aligned}
& \Gamma_{22}^{1}=-x^{1}, \Gamma_{33}^{1}=-x^{1}\left(\sin x^{2}\right)^{2}, \Gamma_{12}^{2}=\frac{1}{x^{1}}=\Gamma_{13}^{3}, \Gamma_{23}^{3}=\cot x^{2}, \Gamma_{33}^{2}=-\sin x^{2} \cos x^{2}, \\
& R_{2332}=-\left(x^{1} \sin x^{2}\right)^{2}
\end{aligned}
$$

and the components which can be obtained from these by the symmetry properties. Using the above relations, we can find the non-vanishing components of Ricci tensor as follows:

$$
\begin{aligned}
& S_{22}=-1, \\
& S_{33}=-\left(\sin x^{2}\right)^{2} .
\end{aligned}
$$

Also it can be easily found that the scalar curvature of the manifold is non-zero and is given by $r=-\frac{2}{\left(x^{1}\right)^{2}} \neq 0$. Therefore $R^{4}$ with the considered metric is a Riemannian manifold $\left(M^{4}, g\right)$ of non-vanishing scalar curvature. We shall now show that this $M^{4}$ is a $P(G Q E)_{4}$ i.e., it satisfies the defining relation (1.3). Let us now consider the associated scalars, associated 1-forms and structure tensor as follows:

$$
\begin{aligned}
& \alpha=-\frac{1}{\left(x^{1}\right)^{2}}, \beta=\frac{1}{\left(x^{1}\right)^{2}}, \gamma=\frac{1}{\left(x^{1}\right)^{2}}, \delta=\frac{2}{\left(x^{1}\right)^{2}}, \\
& A_{i}(x)=x^{1} \text { for } i=2, \\
& =0 \quad \text { otherwise, }
\end{aligned}
$$




$$
\begin{gathered}
B_{i}(x)=1 \quad \text { for } i=2, \\
=x^{1} \quad \text { for } i=3, \\
=0 \quad \text { otherwise, } \\
D_{i j}(x)=\frac{1}{2} \quad \text { for } i=j=1,4, \\
=-\frac{1}{2} \quad \text { for } i=j=2,3, \\
=-x^{1} \quad \text { for } i=2, j=3, \\
=0 \quad \text { otherwise }
\end{gathered}
$$

at any point $x \in M$. In our $M^{4}$, (1.3) reduces with these associated scalars, 1 -forms and structure tensor to the following equations:

(i) $S_{11}=\alpha g_{11}+\beta A_{1} A_{1}+\gamma B_{1} B_{1}+\delta D_{11}$,

(ii) $S_{22}=\alpha g_{22}+\beta A_{2} A_{2}+\gamma B_{2} B_{2}+\delta D_{22}$,

(iii) $S_{23}=\alpha g_{23}+\beta A_{2} A_{3}+\gamma B_{2} B_{3}+\delta D_{23}$,

(iv) $S_{33}=\alpha g_{33}+\beta A_{3} A_{3}+\gamma B_{3} B_{3}+\delta D_{33}$,

(v) $S_{44}=\alpha g_{44}+\beta A_{4} A_{4}+\gamma B_{4} B_{4}+\delta D_{44}$

since for the cases other than (i) - (v) the components of each term of (1.3) vanishes identically and the relation (1.3) holds trivially. Now from (7.2)-(7.5) we get the following relations for the right hand side (R.H.S.) and left hand side (L.H.S.) of (i):

$$
\text { R.H.S. of (i) }=\alpha g_{11}+\beta A_{1} A_{1}+\gamma B_{1} B_{1}+\delta D_{11}=0=S_{11}=\text { L.H.S. of (i). }
$$

Again

$$
\begin{aligned}
& \text { R.H.S. of (ii) }=\alpha g_{22}+\beta A_{2} A_{2}+\gamma B_{2} B_{2}+\delta D_{22}=-1=S_{22}=\text { L.H.S. of (v), } \\
& \text { R.H.S. of (iv) }=\alpha g_{33}+\beta A_{3} A_{3}+\gamma B_{3} B_{3}+\delta D_{33}=-\left(\sin x^{2}\right)^{2}=S_{33}=\text { L.H.S. of (iv). }
\end{aligned}
$$

By a similar argument as in (i), (ii) and (iv) it can be shown that the relations (iii) and (v) are true. Therefore, $\left(M^{4}, g\right)$ is a $P(G Q E)_{4}$ which is neither $(Q E)_{4}$ nor $G(Q E)_{4}$. Hence we can state the following:

Theorem 7.1. Let $\left(M^{4}, g\right)$ be a Riemannian manifold endowed with the metric given by

$$
\begin{aligned}
d s^{2}= & g_{i j} d x^{i} d x^{j}=\left(d x^{1}\right)^{2}+\left(x^{1}\right)^{2}\left(d x^{2}\right)^{2}+\left(x^{1} \sin x^{2}\right)^{2}\left(d x^{3}\right)^{2}+\left(d x^{4}\right)^{2}, \\
& (i, j=1,2, \ldots, 4),
\end{aligned}
$$

where $x^{1} \neq 0$ and $0<x^{2}<\frac{\pi}{2}$. Then $\left(M^{4}, g\right)$ is a pseudo generalized quasi-Einstein manifold of non-vanishing scalar curvature which is neither quasi-Einstein nor generalized quasi-Einstein.

Example 2. We define a Riemannian metric on the 4-dimensional real number space $R^{4}$ by the formula

$$
\begin{aligned}
d s^{2}= & g_{i j} d x^{i} d x^{j}=e^{x^{1}}\left(d x^{1}\right)^{2}+e^{x^{2}}\left(d x^{2}\right)^{2}+e^{x^{3}}\left(d x^{3}\right)^{2}+\left(\sin x^{3}\right)^{2}\left(d x^{4}\right)^{2}, \\
& (i, j=1,2, \ldots, 4),
\end{aligned}
$$


where $\frac{\pi}{4}<x^{3}<\frac{\pi}{2}$. Then the only non-vanishing components of the Christoffel symbols and the curvature tensor are

$$
\begin{aligned}
& \Gamma_{11}^{1}=-\frac{1}{2}=\Gamma_{22}^{2}=\Gamma_{33}^{3}, \quad \Gamma_{34}^{4}=\cot x^{3}, \quad \Gamma_{44}^{3}=-e^{-x^{3}} \sin x^{3} \cos x^{3}, \\
& R_{3443}=1+\frac{3}{2} \sin x^{3} \cos x^{3}-3\left(\cos x^{3}\right)^{2}
\end{aligned}
$$

and the components which can be obtained from these by the symmetry properties. Using the above relations, we can find the non-vanishing components of Ricci tensor as follows:

$$
\begin{aligned}
& S_{33}=1+\frac{3}{2} \cot x^{3}-2\left(\cot x^{3}\right)^{2}, \\
& S_{44}=e^{-x^{3}}\left[1+\frac{3}{2} \sin x^{3} \cos x^{3}-3\left(\cos x^{3}\right)^{2}\right] .
\end{aligned}
$$

Also it can be easily shown that the scalar curvature of the manifold is non-vanishing. Therefore $R^{4}$ with the considered metric is a Riemannian manifold $\left(M^{4}, g\right)$ of non-vanishing scalar curvature. We shall now show that this $M^{4}$ is a $P(G Q E)_{4}$ i.e., it satisfies the defining condition (1.3). Let us now consider the associated scalars, associated 1-forms and structure tensor as follows:

$$
\begin{aligned}
& \alpha=-e^{-x^{3}}\left(\cot x^{3}\right)^{2}, \beta=3 e^{-x^{3}} \cot x^{3}, \gamma=e^{-x^{3}}, \delta=e^{-x^{3}}\left(\cot x^{3}\right)^{2}, \\
& A_{i}(x)=\sqrt{\frac{3 e^{x^{3}}+2 e^{x^{1}} \cot x^{3}}{6}} \text { for } i=3 \text {, } \\
& =\sqrt{\frac{3}{2}\left(\cot x^{3}\right)^{2}+1} \text { for } i=4, \\
& =0 \text { otherwise, } \\
& B_{i}(x)=\sqrt{e^{x^{3}}-\left(\cot x^{3}\right)^{2}} \text { for } i=3 \text {, } \\
& =0 \text { otherwise, } \\
& D_{i j}(x)=e^{x^{1}} \quad \text { for } i=j=1 \text {, } \\
& =e^{x^{2}} \text { for } i=j=2 \text {, } \\
& =-e^{x^{1}} \text { for } i=j=3 \text {, } \\
& =\sqrt{\frac{\left[e^{x^{3}}-\left(\cot x^{3}\right)^{2}\right]\left[3 e^{x^{3}}+2 e^{x^{1}} \cot x^{3}\right]}{6}} \text { for } i=3, j=4, \\
& =e^{-x^{2}} \text { for } i=j=4 \text {, } \\
& =0 \text { otherwise }
\end{aligned}
$$

at any point $x \in M$. In our $M^{4}$, (1.3) reduces with these associated scalars, 1-forms and structure tensor to the following equations 
(i) $S_{11}=\alpha g_{11}+\beta A_{1} A_{1}+\gamma B_{1} B_{1}+\delta D_{11}$,

(ii) $S_{22}=\alpha g_{22}+\beta A_{2} A_{2}+\gamma B_{2} B_{2}+\delta D_{22}$,

(iii) $S_{33}=\alpha g_{33}+\beta A_{3} A_{3}+\gamma B_{3} B_{3}+\delta D_{33}$,

(iv) $S_{34}=\alpha g_{34}+\beta A_{3} A_{4}+\gamma B_{3} B_{4}+\delta D_{34}$,

(v) $S_{44}=\alpha g_{44}+\beta A_{4} A_{4}+\gamma B_{4} B_{4}+\delta D_{44}$,

since for the cases other than (i) - (v) the components of each term of (1.3) vanishes identically and the relation (1.3) holds trivially. By virtue of (7.7)-(7.10) we get the following relations for the right hand side (R.H.S.) and left hand side (L.H.S.) of (iii):

$$
\begin{aligned}
\text { R.H.S. of (iii) } & =\alpha g_{33}+\beta A_{3} A_{3}+\gamma B_{3} B_{3}+\delta D_{33}=1+\frac{3}{2} \cot x^{3}-2\left(\cot x^{3}\right)^{2} \\
& =S_{33}=\text { L.H.S. of (iii). }
\end{aligned}
$$

By a similar argument as in (iii) it can be shown that the relations (i), (ii), (iv) and (v) are true. Therefore, $\left(M^{4}, g\right)$ is a $P(G Q E)_{4}$ which is neither $(Q E)_{4}$ nor $G(Q E)_{4}$. Thus we can state the following:

Theorem 7.2. Let $\left(M^{4}, g\right)$ be a Riemannian manifold endowed with the metric given by

$$
d s^{2}=g_{i j} d x^{i} d x^{j}=e^{x^{1}}\left(d x^{1}\right)^{2}+e^{x^{2}}\left(d x^{2}\right)^{2}+e^{x^{3}}\left(d x^{3}\right)^{2}+\left(\sin x^{3}\right)^{2}\left(d x^{4}\right)^{2},(i, j=1,2, \ldots, 4)
$$

where $\frac{\pi}{4}<x^{3}<\frac{\pi}{2}$. Then $\left(M^{4}, g\right)$ is a pseudo generalized quasi-Einstein manifold of nonvanishing scalar curvature which is neither quasi-Einstein nor generalized quasi-Einstein.

Example 3. We define a Riemannian metric on the 4-dimensional real number space $R^{4}$ by the formula

$$
d s^{2}=g_{i j} d x^{i} d x^{j}=(1+2 p)\left[\left(d x^{1}\right)^{2}+\left(d x^{2}\right)^{2}+\left(d x^{3}\right)^{2}+\left(d x^{4}\right)^{2}\right],(i, j=1,2, \ldots, 4)
$$

where $p=\frac{e^{x^{1}}}{\rho^{2}}$ and $\rho$ is a non-zero constant. Then the only non-vanishing components of the Christoffel symbols and the curvature tensor are

$$
\begin{aligned}
& \Gamma_{22}^{1}=\Gamma_{33}^{1}=\Gamma_{44}^{1}=-\frac{p}{1+2 p}, \Gamma_{11}^{1}=\Gamma_{12}^{2}=\Gamma_{13}^{3}=\Gamma_{14}^{4}=\frac{p}{1+2 p}, \\
& R_{1221}=R_{1331}=R_{1441}=\frac{p}{1+2 p}
\end{aligned}
$$

and the components which can be obtained from these by the symmetry properties. Using the above relations, we can find the non-vanishing components of Ricci tensor as follows:

$$
\begin{aligned}
& S_{11}=\frac{3 p}{(1+2 p)^{2}}, \\
& S_{22}=\frac{p}{(1+2 p)^{2}}, \\
& S_{33}=\frac{p}{(1+2 p)^{2}}, \\
& S_{44}=\frac{p}{(1+2 p)^{2}} .
\end{aligned}
$$


Also it can be easily shown that the scalar curvature of the manifold is non-vanishing. Therefore $R^{4}$ with the considered metric is a Riemannian manifold $\left(M^{4}, g\right)$ of non-vanishing scalar curvature. We shall now show that this $M^{4}$ is a $P(G Q E)_{4}$ i.e., it satisfies the defining condition (1.3). Let us now consider the associated scalars, associated 1-forms and structure tensor as follows:

$$
\begin{aligned}
& \alpha=\frac{p}{(1+2 p)^{3}}, \beta=\frac{4 p}{(1+2 p)^{3}}, \gamma=-\frac{2 p}{(1+2 p)^{3}}, \delta=\frac{p}{(1+2 p)^{3}}, \\
& A_{i}(x)=\sqrt{p} \text { for } i=1 \text {, } \\
& =\frac{1}{2} \text { for } i=3 \text {, } \\
& =\frac{p}{2} \text { for } i=4 \text {, } \\
& =0 \text { otherwise, } \\
& B_{i}(x)=\frac{p}{\sqrt{2}} \text { for } i=2 \text {, } \\
& =0 \text { otherwise, } \\
& D_{i j}(x)=1 \text { for } i=j=1 \text {, } \\
& =-2 \sqrt{p} \text { for } i=1, j=3 \text {, } \\
& =-2 p^{\frac{3}{2}} \text { for } i=1, j=4 \text {, } \\
& =p^{2} \text { for } i=j=2 \text {, } \\
& =-1 \text { for } i=j=3 \text {, } \\
& =-p \text { for } i=3, j=4 \text {, } \\
& =-p^{2} \text { for } i=j=4 \text {, } \\
& =0 \text { otherwise }
\end{aligned}
$$

at any point $x \in M$. In our $M^{4}$, scalars, 1 -forms and structure tensor to the following equations

(i) $S_{11}=\alpha g_{11}+\beta A_{1} A_{1}+\gamma B_{1} B_{1}+\delta D_{11}$,

(ii) $S_{13}=\alpha g_{13}+\beta A_{1} A_{3}+\gamma B_{1} B_{3}+\delta D_{13}$,

(iii) $S_{14}=\alpha g_{14}+\beta A_{1} A_{4}+\gamma B_{1} B_{4}+\delta D_{14}$,

(iv) $S_{22}=\alpha g_{22}+\beta A_{2} A_{2}+\gamma B_{2} B_{2}+\delta D_{22}$,

(v) $S_{33}=\alpha g_{33}+\beta A_{3} A_{3}+\gamma B_{3} B_{3}+\delta D_{33}$,

(vi) $S_{34}=\alpha g_{34}+\beta A_{3} A_{4}+\gamma B_{3} B_{4}+\delta D_{34}$,

(vii) $S_{44}=\alpha g_{44}+\beta A_{4} A_{4}+\gamma B_{4} B_{4}+\delta D_{44}$,

since for the cases other than (i) -(vii) the components of each term of (1.3) vanishes identically and the relation (1.3) holds trivially. By virtue of (7.12)-(7.15) we get the following relations for the right hand side (R.H.S.) and left hand side (L.H.S.) of (i):

$$
\text { R.H.S. of (i) }=\alpha g_{11}+\beta A_{1} A_{1}+\gamma B_{1} B_{1}+\delta D_{11}=\frac{3 p}{(1+2 p)^{2}}=S_{11}=\text { L.H.S. of (i). }
$$


By a similar argument as in (i) it can be shown that the relations (ii) -(vii) are true. Therefore, $\left(M^{4}, g\right)$ is a $P(G Q E)_{4}$ which is neither $(Q E)_{4}$ nor $G(Q E)_{4}$. Thus we can state the following:

Theorem 7.3. Let $\left(M^{4}, g\right)$ be a Riemannian manifold endowed with the metric given by

$$
d s^{2}=g_{i j} d x^{i} d x^{j}=(1+2 p)\left[\left(d x^{1}\right)^{2}+\left(d x^{2}\right)^{2}+\left(d x^{3}\right)^{2}+\left(d x^{4}\right)^{2}\right],(i, j=1,2, \ldots, 4)
$$

where $p=\frac{e^{x^{1}}}{\rho^{2}}$ and $\rho$ is a constant. Then $\left(M^{4}, g\right)$ is a pseudo generalized quasi-Einstein manifold of non-vanishing scalar curvature which is neither quasi-Einstein nor generalized quasiEinstein.

\section{References}

[1] Chaki, M. C. and Maity, R. K. : On quasi-Einstein manifolds, Publ. Math. Debrecen, 57(2000), 297306.

[2] Chen, B. Y. and Yano, K. : Hypersurfaces of conformally flat spaces, Tensor N. S., 26(1972), 318-322.

[3] De, U. C. and Ghosh, G. C. : On generalized quasi-Einstein manifolds, Kyungpook Math. J., 44(2004), 607-615.

[4] Novello, M. and Reboucas, M. J. : The stability of a rotating universe, The Astrophysics Journal, 225(1978), 719-724.

[5] O’Neill, B. : Semi-Riemannian Geometry, Academic Press, 1983.

[6] Spivak, M. : A comprehensive Introduction to Differential Geometry, Publish and Perish, Vol. I(1970).

[7] Yano, K. : Integral formulas in Riemannian Geometry, Marcel Dekker, New York, 1970.

Department of Mathematics, University of Burdwan, Golapbag, Burdwan-713104, West Bengal, India. E-mail: aask2003@yahoo.co.in, aask@epatra.com

Department of Mathematics, University of Burdwan, Golapbag, Burdwan-713104, West Bengal, India. 